\title{
A Sparse Version of the Ridge Logistic Regression for Large-Scale Text Categorization
}

\author{
Sujeevan Aseervatham ${ }^{\mathrm{a}, *}$, Anestis Antoniadis ${ }^{\mathrm{b}}$, Eric Gaussier $^{\mathrm{a}}$, Michel Burlet ${ }^{\mathrm{c}}$, \\ Yves Denneulin ${ }^{\mathrm{d}}$ \\ ${ }^{a}$ LIG - Université Joseph Fourier, 385, rue de la Bibliothèque, BP 53, F-38041 Grenoble \\ Cedex 9, France \\ ${ }^{b}$ LJK - Université Joseph Fourier, BP 53, F-38041 Grenoble Cedex 9, France \\ ${ }^{c}$ Lab. Leibniz - Université Joseph Fourier, 46 Avenue Félix Viallet, F-38031 Grenoble \\ Cedex 1, France \\ ${ }^{d}$ LIG - ENSIMAG, 51 avenue Jean Kuntzmann, F-38330 Montbonnot Saint Martin, \\ France
}

\begin{abstract}
The ridge logistic regression has successfully been used in text categorization problems and it has been shown to reach the same performance as the Support Vector Machine but with the main advantage of computing a probability value rather than a score. However, the dense solution of the ridge makes its use unpractical for large scale categorization. On the other side, LASSO regularization is able to produce sparse solutions but its performance is dominated by the ridge when the number of features is larger than the number of observations and/or when the features are highly correlated. In this paper, we propose a new model selection method which tries to approach the ridge solution by a sparse solution. The method first computes the ridge solution and then performs feature selection. The experimental evaluations show that our method gives a solution which is a good trade-off between the ridge and LASSO solutions.

Keywords: Logistic Regression, Model Selection, Text Categorization, Large Scale Categorization

\footnotetext{
*Corresponding author, Tel.: +33(0)476514515; Fax: +33(0)476446675

Email addresses: Sujeevan.Aseervatham@imag.fr (Sujeevan Aseervatham) Anestis.Antoniadis@imag.fr (Anestis Antoniadis), Eric.Gaussier@imag.fr (Eric Gaussier), Michel.Burlet@imag.fr (Michel Burlet), Yves.Denneulin@imag.fr (Yves Denneulin)
} 


\section{1. Introduction}

2 The automatic text categorization problem consists in assigning, according 3 to its content, a textual document to one or more relevant predefined categories.

4 Given a training dataset, where the documents have been manually labeled, the

5 problem lies in inducing a function $f$, from the training data, which can then

6 be used to classify documents. Machine learning algorithms are used to find

7 the optimal $f$ by solving a minimization problem which can be stated as the

8 minimization of the cost of misclassification over the training dataset (Empirical

9 Risk Minimization).

10 In order to use numerical machine learning algorithm, the Vector Space

11 Model is commonly used to represent a textual documents by a simple term-

12 frequency vector (Salton et al., 1975). This representation produces datasets in

13 which 1) the number of features is often larger than the number of documents,

14 2) the vectors are very sparse, i.e., a lot of features are set to zero and 3) the

15 features are highly correlated (due to the nature of natural languages). More-

16 over, real-life datasets tend to be larger and larger which makes the automatic

17 categorization process complicated and leads to scalability problems. As long as

18 the datasets only grow in terms of the number of observations, the problem can

19 be tackled by distributing the computation over a network of processors (Chu

20 et al., 2006). However, when the number of features becomes larger than the

21 number of observations, machine learning techniques tend to perform poorly

22 due to overfitting, i.e., the model performs well on the training set but poorly

23 on any other set. To prevent overfitting, the complexity of the model must be

24 controlled during the training process, through model selection techniques. In

25 the Support Vector Machine (SVM) algorithm (Vapnik, 1995), the model com-

26 plexity is given by the $\mathrm{VC}$-dimension, which is the maximum number of vectors,

$\mathbf{2 7}$ for any combination of labels, that can be shattered by the model. SVMs rely

28 on the Structural Risk Minimization (SRM) principle, which not only aims at

29 minimizing the empirical risk (Empirical Risk Minimization - ERM) but also

30 the VC-dimension. SVMs have been used for text categorization and their per- 
31 formance is among the best ones obtained so far (Joachims, 1998).

32 The VC-dimension remaining unknown for many functions, the SRM is dif-

33 ficult to implement. Another model selection, widely used, is to minimize both

34 the ERM and a regularization term: $\lambda \Omega[f]$ where $\lambda$ is a penalty factor, $\Omega[f]$

35 a convex non-negative regularization term and $f$ the model. For linear func-

36 tions: $f(\mathbf{x})=\langle\mathbf{w}, \mathbf{x}\rangle+b$, the regularization term is often defined as $\Omega[f]=\|\mathbf{w}\|_{p}$

37 where $\|\cdot\|_{p}$ is the $L_{p}$-norm (Hoerl and Kennard, 1970; Tibshirani, 1994; Zou and

38 Hastie, 2005). This has the effect of smoothing $f$ and reducing its generaliza-

39 tion error. The use of the $L_{2}$-norm is known as the ridge penalization, whereas

40 the use of the $L_{1}$-norm as the LASSO penalization, which has the property of

41 simultaneously doing shrinkage and feature selection.

42 In this paper, we focus on penalized logistic regression. Logistic regression 43 has the main advantage of computing a probability value rather than a score,

44 as for the SVM. Furthermore, the ridge logistic regression has been shown to

45 reach the same performance as the SVM on standard text categorization prob-

46 lems (Zhang and Oles, 2001). Nevertheless, it produces a dense solution which

47 cannot be used for large scale categorization. In (Genkin et al., 2007), the

48 LASSO logistic regression was used to obtain a sparse solution. However, when

49 the number of features is larger than the number of observations and/or when

50 the features are correlated, the ridge penalization performance dominates the

51 LASSO one (Zou and Hastie, 2005). Taking into account these observations, we

52 propose a new model selection which produces a sparse solution by approaching

53 the ridge solution.

54 The rest of the paper is organized as follows: in the next section we discuss

55 related works; we then describe, in section 3, our model selection approach

56 before reporting, in section 4, our experimental results; section 5 concludes the

57 paper. 


\section{2. Related work}

59 In (le Cessie and van Houwelingen, 1992), the authors have shown how ridge 60 penalization can be used to improve the logistic regression parameter estimates

61 in the cases where the number of features is larger than the number of obser-

62 vations or when the variables are highly correlated. They have applied ridge

63 logistic regression on DNA data and have obtained good results with stable pa-

64 rameters. More recently, the ridge logistic regression was used in (Zhang and

65 Oles, 2001) on the text categorization problem where the data are sparse and

66 the number of features is larger than the number of observations. The authors

67 have proposed several algorithms, which take advantage of the sparsity of the

68 data, to solve efficiently the ridge optimization problem. The experimental re-

69 sults show that the $L_{2}$ logistic regression reaches the same performance as the

70 SVM. Although the ridge method allows to select a more stable model by doing

71 continuous shrinkage, the produced solution is dense and thus not appropriate

$\mathbf{7 2}$ for large and sparse data such as textual data.

73 The LASSO regularization $\left(L_{1}\right.$-norm) has been introduced in (Tibshirani, 1994).

74 The author shows, for linear regression, that the $L_{1}$ penalization can not only do

75 continuous shrinkage but has also the property of doing automatic variable se-

76 lection simultaneously which means that the $L_{1}$ solution is sparse. In (Genkin

77 et al., 2007), an optimization algorithm based on (Zhang and Oles, 2001) is

78 presented for Ridge and LASSO logistic regressions in the context of text cate-

79 gorization. According to their experiments, the lasso penalization gives slightly

80 better results than the ridge penalization in terms of the macro-averaged- $F_{1}$

81 measure (the micro-averaged results are not given). It has been shown in (Efron

82 et al., 2004; Tibshirani, 1994; Zou and Hastie, 2005) that the performance of

83 the LASSO is dominated by the ridge in the following cases (we denote by $p$ the

84 number of features and by $n$ the number of observations):

$85 \quad-p>n$ : the LASSO will only select at most $n$ features,

86 - the features are highly correlated: the LASSO will select only one feature 87 among the correlated features. 
88 To tackle the limitations of the LASSO, the Elastic net method has been pro-

89 posed in (Zou and Hastie, 2005) which tries to capture the best of both $L_{1}$ and

$90 \quad L_{2}$ penalizations. The Elastic net uses both $L_{1}$ and $L_{2}$ regularization in the lin-

91 ear regression problem. The authors show that the $L_{2}$ regularization term can

92 be reformulated by adding $p$ artificial input data such that each artificial data $i$

93 has only the $i^{\text {th }}$ component non-null set to $\sqrt{\lambda_{2}}$ where $\lambda_{2}$ is the $L_{2}$ regularization

94 hyperparameter. This reformulation, which leads to a LASSO problem, relies

95 on the particular form of the least square term, and cannot be extended to the

96 logistic regression problem. Furthermore, as the $L_{1}$ and $L_{2}$ regularizations are

97 done simultaneously, it is unclear how the solution of the Elastic net approaches

98 the $L_{2}$ solution. In (Zhao and Yu, 2006), the model consistency of LASSO is

99 studied for linear regression and it is shown that the consistency of LASSO

100 depends on the regularization parameter. In (Bach, 2008), the author proves

101 that for a regularization parameter decay factor of $\frac{1}{\sqrt{n}}$, a consistent model can

102 be obtained by applying LASSO on bootstrap samples and by selecting only

103 the intersecting features. Nevertheless, using LASSO on bootstrap samples is

104 a time consuming process. Moreover, since this method is based on LASSO, it

105 also fails to induce a good model when the variables are correlated.

\section{3. Selected Ridge Logistic Regression}

107 The logistic regression model is part of the Generalized Linear Model (GLM)

108 family (Hastie and Tibshirani, 1990; Mccullagh and Nelder, 1989). The GLM

109 is a family of models, parametrized by $\beta$, which associate a target variable $y$ to

110 an input data $\mathbf{x}\left(x \in \mathbb{R}^{p}\right)$ according to the relation $\beta \cdot \mathbf{x}=g(y)$ where $g$ is a link

111 function and $\beta \in \mathbb{R}^{p}$. For simplicity, we represent any linear function $\beta^{\prime} \cdot \mathbf{x}^{\prime}+\beta_{0}^{\prime}$

112 by $\beta \cdot \mathbf{x}$, where $\mathbf{x}$ is $\mathbf{x}^{\prime}$ with an extra dimension set to 1 , and $\beta$ is $\beta^{\prime}$ with an

113 extra dimension set to $\beta_{0}^{\prime}$. The logistic regression model is obtained by using

114 a logit function $g(y)=\frac{P(y \mid \beta, \mathbf{x})}{1-P(y \mid \beta, \mathbf{x})}$. When $y \in\{-1,1\}$, the logistic regression

115 model can be written as: 


$$
P(y=1 \mid \beta, \mathbf{x})=\frac{1}{1+\exp (-\beta \cdot \mathbf{x})}
$$

$116 \beta$ can be obtained by maximizing the $\log$-likelihood over the training set $\mathcal{D}=$ $117\left\{\left(\mathbf{x}_{\mathbf{1}}, y_{1}\right), \ldots,\left(\mathbf{x}_{\mathbf{n}}, y_{n}\right)\right\}$. However, in order to obtain a strictly convex optimiza118 tion problem and to avoid overfitting, a Tikhonov regularization term (Hoerl 119 and Kennard, 1970) is added, leading to the following ridge logistic regression 120 problem:

$$
\beta^{*}=\underset{\beta}{\operatorname{argmin}} \underbrace{\sum_{i=1}^{n} \log \left(1+\exp \left(-y_{i} \beta \cdot \mathbf{x}_{\mathbf{i}}\right)\right)+\lambda\|\beta\|_{2}^{2}}_{l(\beta)}
$$

121 where $\lambda$ is a strictly positive scalar. Adding a ridge regularization term is 122 equivalent, in a Bayesian framework, to using a Gaussian prior on each com123 ponent of $\beta$, under the assumption that the components are independent, i.e. $124 P(\beta)=\prod_{j} P\left(\beta_{j}\right)$ with $P\left(\beta_{j}\right) \sim \mathcal{N}\left(0, \frac{1}{2 \lambda}\right)$.

125 Several algorithms have been proposed in the literature to solve the opti126 mization problem in 2 (Friedman et al., 2008; Minka, 2003). In (Genkin et al., 127 2007), an efficient algorithm, based on the one presented in (Zhang and Oles, 128 2001), is proposed to solve problems with sparse data, such as text documents.

129 However, the ridge regression solution is a dense vector which can hardly be 130 used in large scale categorization where hundreds of thousand features are used.

131 The problem we face is thus the one of finding $\hat{\beta}$ such that:

$1321 . \hat{\beta}$ is close to $\beta^{*}$ and thus behaves well, ie $l(\hat{\beta}) \simeq l\left(\beta^{*}\right)$;

$1332 . \hat{\beta}$ is a sparse solution and thus can be used on large datasets.

134 The second order Taylor series expansion on $l(\beta)$ around $\beta^{*}$ leads to:

$$
\begin{aligned}
l(\beta) \simeq & l\left(\beta^{*}\right)+\left(\beta-\beta^{*}\right)^{T} \nabla l\left(\beta^{*}\right) \\
& +\frac{1}{2}\left(\beta-\beta^{*}\right)^{T} H_{l}\left(\beta^{*}\right)\left(\beta-\beta^{*}\right) \\
= & l\left(\beta^{*}\right)+\frac{1}{2}\left(\beta-\beta^{*}\right)^{T} H_{l}\left(\beta^{*}\right)\left(\beta-\beta^{*}\right)
\end{aligned}
$$


135 where $\nabla l\left(\beta^{*}\right)$ and $H_{l}\left(\beta^{*}\right)$ are respectively the gradient and the Hessian of $l(\beta)$

136 at $\beta^{*}$ and where the equality derives from the fact that for $\beta^{*}$, the ridge solution,

137 the gradient vanishes. Hence, obtaining a $\hat{\beta}$ yielding a value for $l$ close to the

138 one of $\beta^{*}$ while being sparse can be achieved by solving the following strictly

139 convex optimization problem:

$$
\hat{\beta}=\underset{\beta}{\operatorname{argmin}}\left(\beta-\beta^{*}\right)^{T} H_{l}\left(\beta^{*}\right)\left(\beta-\beta^{*}\right)+\alpha\|\beta\|_{1}
$$

140 The $L_{1}$ regularization term, used to ensure sparsity, corresponds, in the Bayesian

141 framework, to the Laplace distribution prior on the components of $\beta$ : $P\left(\beta_{i}\right) \sim$

142 Laplace $\left(0, \frac{1}{\alpha}\right)$ with $\alpha$ a strictly positive scalar. We refer to the above approach

143 as the Selected Ridge Logistic Regression method.

144 The so-called bag-of-words representation used in most text classification

145 methods assumes independence between words in documents ${ }^{1}$. Such an inde-

146 pendence assumption naturally leads to assuming that the components of $\beta$ are

147 independent of one another, and thus that the Hessian $H_{l}\left(\beta^{*}\right)$ is diagonal. We

148 make this assumption in the remainder of the paper. In this case, an analytical

149 solution to equation 4 can be obtained. Indeed, equation 4 can be rewritten as:

$$
\hat{\beta}=\underset{\beta}{\operatorname{argmin}} \sum_{i=1}^{p}\left(\beta_{i}-\beta_{i}^{*}\right)^{2} H_{i}\left(\beta^{*}\right)+\alpha\|\beta\|_{1}
$$

150 with

$$
H_{i}(\beta)=\frac{\partial^{2} l(\beta)}{\partial \beta_{i}^{2}}=\sum_{j=1}^{n} \frac{x_{j i}{ }^{2} \exp \left(-y_{j} \beta \cdot \mathbf{x}_{\mathbf{j}}\right)}{\left(1+\exp \left(-y_{j} \beta \cdot \mathbf{x}_{\mathbf{j}}\right)\right)^{2}}+2 \lambda
$$

151 Thus, the overall optimization problem can be solved component by component:

$$
\hat{\beta}_{i}=\underset{\beta_{i}}{\operatorname{argmin}}\left(\beta_{i}-\beta_{i}^{*}\right)^{2} H_{i}\left(\beta^{*}\right)+\alpha\left|\beta_{i}\right|
$$

152 and its solution is given by theorem 3.1 .

\footnotetext{
${ }^{1}$ (Joachims, 2002) for example recommends to use linear kernels, and not polynomial or Gaussian ones, for text classification.
} 
153 Theorem 3.1. The solution, $\hat{\beta}_{i}$, of the minimization problem in 7 is given by:

$$
\hat{\beta}_{i}= \begin{cases}\beta_{i}^{*}-\frac{\alpha}{2 H_{i}\left(\beta^{*}\right)} & \text { if } \beta_{i}^{*}>\frac{\alpha}{2 H_{i}\left(\beta^{*}\right)} \\ \beta_{i}^{*}+\frac{\alpha}{2 H_{i}\left(\beta^{*}\right)} & \text { if } \beta_{i}^{*}<-\frac{\alpha}{2 H_{i}\left(\beta^{*}\right)} \\ 0 & \text { otherwise }\end{cases}
$$

154 (note that $\hat{\beta}_{i}=0$ if $H_{i}\left(\beta^{*}\right)=0$ ).

155 Proof Let us assume that $\beta_{i}^{*} \geq 0$ and let $g\left(\beta_{i}\right)=\left(\beta_{i}-\beta_{i}^{*}\right)^{2} H_{i}\left(\beta^{*}\right)+\alpha\left|\beta_{i}\right|$.

156 We have: $\forall \beta_{i} \geq 0, g\left(\beta_{i}\right) \leq g\left(-\beta_{i}\right)$, so that $\hat{\beta}_{i} \geq 0$. Setting the derivative of the 157 strictly convex function $g$ wrt $\beta_{i}$ to 0 , one gets:

$$
\begin{aligned}
\beta_{i}^{+} & =\underset{\beta_{i}>0}{\operatorname{argmin}} g\left(\beta_{i}\right) \\
& = \begin{cases}\beta_{i}^{*}-\frac{\alpha}{2 H_{i}\left(\beta^{*}\right)} & \text { if } \beta_{i}^{*}>\frac{\alpha}{2 H_{i}\left(\beta^{*}\right)} \\
0 & \text { otherwise. }\end{cases}
\end{aligned}
$$

158 In the case where

$$
\beta_{i}^{*}>\frac{\alpha}{2 H_{i}\left(\beta^{*}\right)}
$$

159 let

$$
\beta_{i}^{*}=\frac{\alpha}{2 H_{i}\left(\beta^{*}\right)}+\epsilon
$$

160 Then, we have:

$$
g(0)=g\left(\beta_{i}^{*}-\frac{\alpha}{2 H_{i}\left(\beta^{*}\right)}\right)+\epsilon^{2} H_{i}\left(\beta^{*}\right)>g\left(\beta_{i}^{*}-\frac{\alpha}{2 H_{i}\left(\beta^{*}\right)}\right)
$$

161 This shows that $\hat{\beta}_{i}=\beta_{i}^{+}$when $\beta_{i}^{*} \geq 0$. The case $\beta_{i}^{*} \leq 0$ is treated in exactly 162 the same way and completes the proof of theorem 3.1.

163 Automatic Setting of the penalty hyperparameter

164 In order to reduce the number of hyperparameters to estimate, one can set the

165 LASSO penalty $\alpha$ to the universal penalty (or universal thresholding). Indeed, 
166 the function to be minimized in 4 can also be interpreted as the penalized loss

167 of a Gaussian vector $\beta$ with mean $\beta^{*}$ and covariance matrix $H_{l}^{-1}\left(\beta^{*}\right)$. For

$168 H_{l}^{-1}\left(\beta^{*}\right)$ bounded in the vicinity of $\beta^{*}$, theorem 4 in (Antoniadis and Fan,

169 2001) applies and defines the universal penalty (or universal thresholding) to be $170 \sqrt{\frac{2 \log (p)}{p}}$, a value which guarantees that the risk function of $\hat{\beta}$ (solution of 4 ) is

171 within a factor of logarithmic order. This leads to the following property:

172 Property 3.1. The universal penalty $\alpha$ for minimizing 4 w.r.t. $\beta$, for $H_{l}^{-1}\left(\beta^{*}\right)$

173 bounded in the vicinity of $\beta^{*}$, is $\sqrt{\frac{2 \log (p)}{p}}$, with $p$ the dimension of $\beta$.

174 The algorithm associated with the above, overall approach can be described as 175 follows.

176 Summary of the approach

177 The Selected Ridge Logistic Regression method is summarized in algorithm 1.

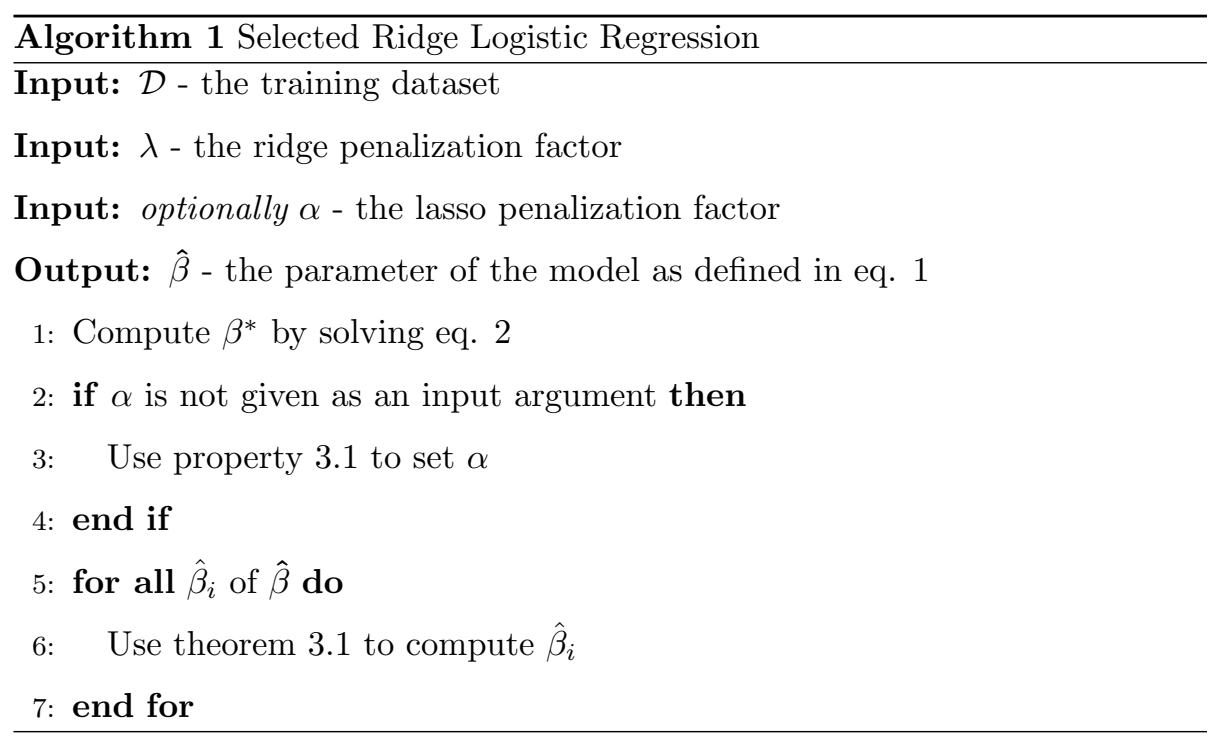

178 Despite the fact that the Selected Ridge method involves the computation of 179 a ridge solution, it is important to note, as we will see in the experimental 180 section, that the training time of the Selected Ridge method is usually shorter 181 than that of the Ridge method. This is due to the fact that the optimal $\lambda$ for 182 both methods are different and, especially in text categorization, the optimal 
$183 \lambda$ for the Selected Ridge method is larger than the optimal $\lambda$ for the Ridge 184 method. For a small $\lambda$ close to zero, the training time of the Ridge method will

185 be important as more iterations will be needed to reach convergence.

\section{Relation to the Fisher Information Matrix}

187 The fisher information matrix $I(\beta)$ is given, for each entry $(i, j)$, by the following 188 equation:

$$
I_{i, j}(\beta)=-\mathbb{E}\left(\frac{\partial^{2} \log P(y \mid \mathbf{x}, \beta)}{\partial \beta_{i} \partial \beta_{j}}\right)
$$

189 Thus, using the empirical Fisher information matrix $\hat{I}\left(\beta^{*}\right)$, we have:

$$
H_{i}\left(\beta^{*}\right)=\hat{I}_{i, i}\left(\beta^{*}\right)+2 \lambda
$$

190 The Fisher information matrix summarizes the average amount of information 191 brought by the data on $\beta$. Hence according to theorem 3.1 and formula 9 , 192 the more information the data brings on $\beta_{i}^{*}$ (ie the higher $\hat{I}_{i, i}\left(\beta^{*}\right)$ ), the higher $193 H_{i}\left(\beta^{*}\right)$ will be and the closer $\hat{\beta}_{i}$ will be to $\beta_{i}^{*}$. In other words, the value obtained

194 through the original ridge regression problem is almost not modified. On the 195 contrary, if the data brings little information on $\beta_{i}^{*}$ (ie $\hat{I}_{i, i}\left(\beta^{*}\right)$ is small), then $196 H_{i}\left(\beta^{*}\right)$ will be small and $\hat{\beta}_{i}$ will be set to zero for a large range of values of $\beta_{i}^{*}$.

197 Thus, sparsity is obtained in the Selected Ridge Regression method by setting to

1980 the dimensions of the ridge solution $\beta^{*}$ which have small values and which 199 are not supported by the data, ie for which $\hat{I}_{i, i}\left(\beta^{*}\right)$ is small. This result reflects 200 the intuition that, in many text categorization problems, only a few words are 201 crucial and usually correspond to the dimensions for which the ridge values are 202 sufficiently large. The development provided here, in particular theorem 3.1, 203 shows that one should discard dimensions for which the ridge value is not larger 204 than, roughly, the inverse of the Fisher information. Thus, the ridge value is not 205 the only parameter one should consider. The information brought by the data 206 on this value plays indeed a crucial role: dimensions with small values strongly 207 supported by the data should be kept in the final solution. 


\section{4. Experimental Results}

209 The proposed model selection method was evaluated over a set of three 210 well-known datasets and one large dataset. The first three datasets are Reuters 211 21578, Ohsumed and 20-NewsGroups (Hersh et al., 1994; Joachims, 1998, 2002).

212 All of these datasets have been widely studied in the text categorization litera213 ture. Reuters $21578^{2}$ is a collection of news on different domains. Ohsumed ${ }^{3}$ is a

214 collection of medical abstracts originally designed for content-based information 215 retrieval, and 20-NewsGroup ${ }^{4}$ a collection of documents written in the context 216 of 20 different news groups. These collections are thus varied in terms of their $\mathbf{2 1 7}$ production and content. The last dataset is a subset of documents taken from 218 the DMOZ website ${ }^{5}$. This DMOZ dataset was collected in order to perform

219 Large-Scale text categorization experiments. The characteristics of the datasets $\mathbf{2 2 0}$ are reported in table 1. This last collection is a collection of web pages, and 221 contains documents of various types (scientific articles, business descriptions, 222 ...) on several domains.

Table 1: Datasets used for the experiments

\begin{tabular}{lccccc} 
Name & $\begin{array}{c}\text { Train. } \\
\text { size }(\mathbf{n})\end{array}$ & Test size & $\begin{array}{c}\text { \#Features } \\
(\mathbf{p})\end{array}$ & \#Categories & Case \\
\hline & & & & & \\
Reuters-21578 & 7770 & 3019 & 6760 & 90 & $p<n\left(\frac{p}{n} \approx 1\right)$ \\
Ohsumed & 6286 & 7643 & 20520 & 23 & $p>n\left(\frac{p}{n} \approx 3\right)$ \\
20-NewsGroups & 12492 & 6246 & 51666 & 20 & $p>n\left(\frac{p}{n} \approx 4\right)$ \\
DMOZ & 20249 & 7257 & 133348 & 3503 & $p>n\left(\frac{p}{n} \approx 6\right)$
\end{tabular}

223 All the datasets have been pre-processed according to the setting defined 224 in (Joachims, 2002), which we briefly describe here. The Vector Space Model 225 (VSM) (Salton et al., 1975) is used to represent the textual documents in a vector

\footnotetext{
${ }^{2}$ http://www.daviddlewis.com/resources/testcollections/reuters21578/

${ }^{3}$ http://ir.ohsu.edu/ohsumed/ohsumed.html

${ }^{4}$ http://people.csail.mit.edu/jrennie/20Newsgroups/

${ }^{5}$ http://www.dmoz.org
} 
226 space. The VSM is also known as the Bag-of-Words (BOW) representation in

$\mathbf{2 2 7}$ which a list of terms is used to define a vector space, each term defining an axis

$\mathbf{2 2 8}$ of the space. A textual document can then be represented as a vector, using for

229 each axis, the corresponding term frequency value. In order to obtain an efficient

$\mathbf{2 3 0}$ vector representation, each document is pre-processed using the following steps:

231 1. Cleaning by removing non-Latin characters, numerical symbols and punc-

232 tuation marks,

233 2. Segmenting terms separated by a white space into a list of words,

234 3. Removing stopwords (using a stopword list),

235 4. Stemming each word using the Porter Stemming algorithm (Porter, 1980).

236 We also used the TF-IDF weighting scheme (Jones, 1988) to give more im-

$\mathbf{2 3 7}$ portance to terms that are frequent in a document (the TF part) and specific

238 to a small number of documents (IDF part). Furthermore, we normalized all

239 document vectors.

240 For multi-class categorization, we use the one-vs-the-rest strategy based on

241 binary logistic regression models. To assign a document to a unique category in

242 mono-label problems, we use the following decision function: $\operatorname{argmax}_{c} P\left(y_{i}=\right.$

$243+1 \mid \beta_{c}, \mathbf{x}_{\mathbf{i}}$ ); if a document can be assigned to several categories (multi-label prob-

244 lems), we assign it to each category $c$ such that $P\left(y_{i}=+1 \mid \beta_{c}, \mathbf{x}_{\mathbf{i}}\right) \geq 0.5$.

245 In the experiments, the $F_{1}$ measure (van Rijsbergen, 1979) is used to evaluate

246 the performance of the classifiers. It is defined as:

$$
F_{1}=\frac{2 \times \mathrm{TP}}{2 \times \mathrm{TP}+\mathrm{FP}+\mathrm{FN}}
$$

247 where TP stands for true positive, FP for false positive and FN for false negative.

248 For multi-class datasets, we used the micro- $F_{1}$ and macro- $F_{1}$ measures. In the

249 micro- $F_{1}$ measure, TP, FP and $\mathrm{FN}$ are summed over each category giving thus

250 an equal weight to each document. In this case, this measure corresponds to

251 the overall precision of the system and provides a measure of the accuracy of

252 the classifier. The macro- $F_{1}$ is the arithmetic mean of $F_{1}$ across the categories, 
253 giving an equal weight to each category. If $F_{1}^{c}$ denotes the $F_{1}$ measure for 254 category $c$, then the micro- $F_{1}$ is defined by:

$$
\text { micro- } \mathrm{F}_{1}=\sum_{c=1}^{K} \frac{N_{c}}{N} F_{1}^{c}
$$

255 where $K$ denotes the number of categories, $N_{c}$ corresponds to the number of 256 documents in category $c$, and $N$ is the total number of documents $\left(N=\sum_{c} N_{c}\right)$. $\mathbf{2 5 7}$ The macro- $F_{1}$ is defined by:

$$
\text { macro- } \mathrm{F}_{1}=\sum_{c=1}^{K} \frac{1}{K} F_{1}^{c}
$$

258 We also report the degree of sparsity for each model. The sparsity is given 259 by :

$$
s=1-\frac{\operatorname{avg} \# \text { features used in the model }}{\text { \#features in the dataset }}
$$

260 A solution based on all the features will thus have a degree of sparsity of 0.

261 Moreover, it is important to note that the penalization parameter was fixed

262 for each algorithm by cross-validation except for the DMOZ subset where the

263 parameter was tuned using a validation set composed of 7256 documents. For

264 the Selected Ridge method, the hyperparameter $\alpha$ in equation 4 was automati265 cally set using property 3.1 .

266 To solve the LASSO and Ridge regression problems, we used the algorithm 267 described in (Genkin et al., 2007). The training and prediction times are given 268 as indications. Since, the calculations were distributed over a set of computers, 269 the given times are the times spent on calculation plus the times consumed by $\mathbf{2 7 0}$ the system (thread swapping, network transfer time, etc.).

271 It is also important to note that in all the results below, the training time 272 of the Selected Ridge method is always shorter than that of the Ridge method.

273 This can be confusing since the Selected Ridge involves the computation of a

274 Ridge solution and, thus, one can expect its training time to be at least equal $\mathbf{2 7 5}$ to that of the Ridge method. Actually, as we said above, this is due to the fact 
276 that the ridge's training time depends on the Ridge regularization parameter $\lambda$.

$\mathbf{2 7 7}$ If $\lambda$ is very close to 0 then the training time will be important as more iterations

278 will be needed to reach convergence. In all our experiments, the optimal $\lambda$ for

279 the Ridge method was always smaller than that for the Selected Ridge method.

280 For example, for the DMOZ dataset in subsection 4.4, the optimal $\lambda$ for Ridge

$\mathbf{2 8 1}$ is 0.0001 (training time: 13299.43s) but the optimal $\lambda$ for the Selected Ridge

282 is 0.001 (training time: 10996.80s). This difference can also be seen in table 6:

283 when the L1-parameter $(\alpha)$ is zero (Selected Ridge=ridge) then the optimal $\lambda$

284 for the Selected Ridge method is 0.0001, but when $\alpha$ is greater than 0 then the

285 optimal $\lambda$ is always 0.001 .

286 4.1. Experiments on Reuters-21587

287 The Reuters-21587 dataset is a collection of newswire articles. Each docu-

288 ment was manually assigned to one or more categories, according to its subject.

289 In this collection, we used the standard "ModApte" split, which provides train-

290 ing and test sets. The results are reported in Table 2. The LASSO and the

291 ridge reach the same level of performance; however, the ridge method yields a

292 dense model whereas the LASSO one only selects $0.0043 \%$ of the features. The

293 feature selection method used on the ridge model (Selected Ridge Regression

294 method) allows to reach the same micro- $F_{1}$ performance than the ridge method,

295 but with a number of features reduced by $95 \%$.

Table 2: Categorization Result on Reuters-21587(ModApte)

\begin{tabular}{lccccc} 
Algorithm & Micro $F_{1}$ & $\begin{array}{c}\text { Macro } \\
F_{1}\end{array}$ & Sparsity & $\begin{array}{c}\text { Training } \\
\text { time (sec) }\end{array}$ & $\begin{array}{c}\text { Pred. time } \\
(\mathbf{s e c})\end{array}$ \\
\hline & & & & & \\
LASSO & 0.8711 & 0.5167 & 0.9957 & 164.07 & 0.44 \\
Ridge & 0.8690 & 0.5099 & 0.0 & 257.96 & 13.20 \\
Selected Ridge & 0.8645 & 0.4563 & 0.9447 & 180.24 & 1.55
\end{tabular}


297 The Ohsumed corpus (Hersh et al., 1994) is a subset of the medical biblio298 graphic database MEDLINE. Each document is a reference of a medical article 299 published in a medical journal. Following the settings defined in (Joachims, 300 1998, 2002), we only kept the first 20,000 references which had abstracts and 301 were published in 1991. This set is split into a training set composed of the first 30210,000 documents and a test set composed of the rest. Only abstracts are used 303 for the categorization task. After the pre-processing, the training set is reduced 304 to 6,286 unique documents and the test set to 7,643. Each document belongs 305 to one or more cardiovascular categories.

306 As shown in table 3, the LASSO method performs well on this dataset; not $\mathbf{3 0 7}$ only it has the best performance in terms of micro and macro- $F_{1}$ but it also 308 gives a very sparse solution. The Selected Ridge method slightly improves the 309 micro- $F_{1}$ performance of the ridge method while removing $88 \%$ of its features.

Table 3: Categorization Result on Ohsumed

\begin{tabular}{lccccc} 
Algorithm & Micro $F_{1}$ & $\begin{array}{c}\text { Macro } \\
F_{1}\end{array}$ & Sparsity & $\begin{array}{c}\text { Training } \\
\text { time (sec) }\end{array}$ & $\begin{array}{c}\text { Pred. time } \\
(\mathbf{s e c})\end{array}$ \\
\hline & & & & & \\
LASSO & 0.6533 & 0.6053 & 0.9800 & 81.16 & 1.83 \\
Ridge & 0.6387 & 0.5897 & 0.0 & 144.06 & 31.20 \\
Selected Ridge & 0.6409 & 0.5802 & 0.8827 & 107.08 & 5.32
\end{tabular}

\section{4.3. Experiments on 20-Newsgroups}

311 The 20-NewsGroups is a collection of emails taken from the Usenet news-

312 groups. Each email is assigned to a unique category according to its topic. The 313 experiment on 20-newsgroups, reported in table 4, clearly shows that the ridge 314 penalization outperforms the LASSO method. In fact, the variable selection

$\mathbf{3 1 5}$ of the LASSO is too aggressive and eliminates interesting features. However, 
316 our variable selection method (Selected Ridge) achieves micro-F1 and macro-F1

317 scores similar to those obtained by the ridge, while relying on only $10 \%$ of the

318 features used in the ridge solution.

Table 4: Categorization Result on 20-NewsGroups

\begin{tabular}{lccccc} 
Algorithm & Micro $F_{1}$ & $\begin{array}{c}\text { Macro } \\
F_{1}\end{array}$ & Sparsity & $\begin{array}{c}\text { Training } \\
\text { time (sec) }\end{array}$ & $\begin{array}{c}\text { Pred. time } \\
(\mathbf{s e c})\end{array}$ \\
\hline & & & & & \\
LASSO & 0.8663 & 0.8644 & 0.9861 & 384.16 & 1.72 \\
Ridge & 0.9038 & 0.9018 & 0.0 & 157.96 & 71.25 \\
Selected Ridge & 0.8966 & 0.8939 & 0.9050 & 136.01 & 7.51
\end{tabular}

319 4.4. Experiments on DMOZ

320 In order to assess the behavior of the different methods in a large scale cat321 egorization setting, we have collected 34,762 html documents from the DMOZ 322 website. DMOZ (www.dmoz.org) is an open directory project that aims to 323 classify the whole web into categories. In the collected dataset, we only used 3243,503 categories and we split the corpus into 3 parts: a training set composed 325 of 20,249 documents, a validation set composed of 7,256 documents and a test 326 set composed of 7,257 documents. The validation set is used to tune the hy327 perparameters. For the pre-processing of the documents, we removed the html 328 tags and the script parts to keep only the text and we applied the standard 329 pre-processing steps described above. For illustration, figure 1 shows a part of a 330 document from the corpus before and after the pre-processing. In this dataset, 331 each document belongs to a unique category.

332 As expected in the case where the number of features is largely greater than 333 the number of documents, the ridge method clearly outperforms LASSO as 334 shown in table 5. However, the ridge solution being dense, the categorization 335 of large sets is a time consuming process which makes the ridge solution inap336 propriate. The LASSO and the Selected Ridge methods both produce a sparse 


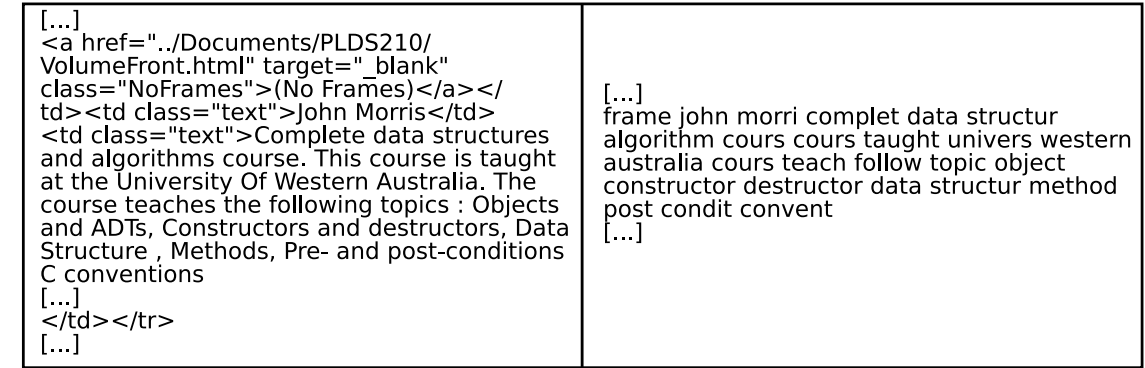

(a) (b)

Figure 1: A part of an html document taken from the DMOZ corpus (a) before and (b) after the pre-processing. The address of the document is http://www.oopweb.com/Algorithms/Files/Algorithms.html and it is referenced in DMOZ as "OOPWeb Algorithms Directory" at http://www.dmoz.org/Computers/Algorithms.

337 solution with a degree of sparsity of $99 \%$. The selected ridge performs better 338 than the LASSO in terms of the micro- $F_{1}$ measure, but however has a macro- $F_{1}$ 339 value slightly lower than the value obtained by LASSO.

Table 5: Categorization Result on DMOZ

\begin{tabular}{lccccc} 
Algorithm & Micro $F_{1}$ & $\begin{array}{c}\text { Macro } \\
F_{1}\end{array}$ & Sparsity & $\begin{array}{r}\text { Training } \\
\text { time (sec) }\end{array}$ & $\begin{array}{r}\text { Pred. time } \\
(\mathbf{s e c})\end{array}$ \\
\hline & & & & & \\
LASSO & 0.2936 & 0.1661 & 0.9999 & 9805.78 & 41.51 \\
Ridge & 0.3434 & 0.2020 & 0.0 & 13299.40 & 31084.90 \\
Selected Ridge & 0.3124 & 0.1586 & 0.9993 & 10996.80 & 42.52
\end{tabular}

340 In table 6, we report the performance of the Selected Ridge method according 341 to the value of the $L_{1}$ penalty term in equation 4 . The results show that 342 property 3.1 provides a good penalty value in terms of trade-off between micro$343 \quad F_{1}$ performance and sparsity. It is also interesting to note that with $\alpha$ set to $34410^{-7}$, one obtains a method yielding results on a par with the ones obtained by 345 the ridge (which provides the best results in terms of both micro- and macro- $F_{1}$ ) 346 while being twice sparser and almost four times faster. 
Table 6: Performance of the Selected Ridge Method on DMOZ according to the penalty value in equation 4 . The results corresponding to the optimal universal penalty value (property 3.1 ) are indicated in bold.

\begin{tabular}{lccccc} 
Penalty $(\alpha)$ & Micro- $F_{1}$ & Macro- $F_{1}$ & Sparsity & $\begin{array}{r}\text { Training } \\
\text { time (sec) }\end{array}$ & $\begin{array}{r}\text { Prediction } \\
\text { time (sec) }\end{array}$ \\
\hline & & & & & \\
1 & 0.1188 & 0.0353 & 0.9999 & 11103.9 & 8.33 \\
0.1 & 0.2604 & 0.1209 & 0.9999 & 11065.8 & 32.81 \\
0.05 & 0.2835 & 0.1343 & 0.9998 & 11325.1 & 39.82 \\
0.03 & 0.2953 & 0.1436 & 0.9997 & 11013.4 & 39.34 \\
0.02 & 0.3040 & 0.1524 & 0.9996 & 11164.6 & 49.07 \\
$\mathbf{0 . 0 1 3 3}$ & $\mathbf{0 . 3 1 2 4}$ & $\mathbf{0 . 1 5 8 6}$ & $\mathbf{0 . 9 9 9 3}$ & $\mathbf{1 0 9 9 6 . 8}$ & $\mathbf{4 2 . 5 2}$ \\
0.01 & 0.3156 & 0.1604 & 0.9992 & 10965.5 & 52.97 \\
$10^{-7}$ & 0.3434 & 0.1949 & 0.5423 & 11090.2 & 8858.31 \\
0 & 0.3434 & 0.2020 & 0.0 & 13299.40 & 31084.90
\end{tabular}

\section{5. Conclusion}

348 As pointed in (Zhao and Yu, 2006): Sparsity or parsimony of statistical mod-

349 els is crucial for their proper interpretations. In this paper, we have proposed a

350 model selection method to "sparsify" the ridge logistic regression solution. This

351 method first solves the classic ridge logistic regression, then sets less informative

352 features with low values to zero, while ensuring that the resulting sparse solution

353 remains in the vicinity of the ridge solution. This latter property is obtained

354 by using a Taylor expansion of the likelihood function around the solution of

355 the ridge, penalized with the $L_{1}$ norm. The experimental text categorization

356 results obtained on well-studied datasets and on a large-scale dataset collected

357 from www.dmoz.org show that our method produces a solution which offers a

358 good trade-off between the performance of the ridge solution and the sparsity

359 of the LASSO solution. In particular, when $p>n$ (the number of features is 
360 greater than the number of observations), our method leads to a sparse version

361 of the ridge which is both accurate (in terms of both micro- and macro- $F_{1}$ ) and

362 fast.

\section{Acknowledgements}

364 This work was partly supported by the LASCAR project, Univ. J. Fourier,

365 Grenoble.

366 Antoniadis, A., Fan, J., 2001. Regularization of Wavelet Approximations. Jour-

367 nal of the American Statistical Association 96, 939-967.

368 Bach, F. R., 2008. Bolasso: model consistent lasso estimation through the boot-

369 strap. In: ICML '08: Proceedings of the 25th international conference on

370 Machine learning. ACM, New York, NY, USA, pp. 33-40.

371 Chu, C.-T., Kim, S. K., Lin, Y.-A., Yu, Y., Ng, G. B. A. Y., Olukotun, K., 372 2006. Map-Reduce for Machine Learning on Multicore. In: Schölkopf, B., 373 Platt, J. C., Hoffman, T. (Eds.), NIPS. MIT Press, pp. 281-288.

374 Efron, B., Hastie, T., Johnstone, L., Tibshirani, R., 2004. Least angle regression. 375 Annals of Statistics 32, 407-499.

376 Friedman, J., Hastie, T., Tibshirani, R., 2008. Regularization paths for gener-

377 alized linear models via coordinate descent. Tech. rep., Dept. of Statistics, 378 Stanford University.

379 Genkin, A., Lewis, D. D., Madigan, D., 2007. Large-Scale Bayesian Logistic 380 Regression for Text Categorization. Technometrics 49, 291-304(14).

381 Hastie, T. J., Tibshirani, R. J., 1990. Generalized Additive Models. Chapman 382 \& Hall.

383 Hersh, W., Buckley, C., Leone, T. J., Hickam, D., 1994. OHSUMED: an in384 teractive retrieval evaluation and new large test collection for research. In: 
386 New York, Inc., New York, NY, USA, pp. 192-201.

387 Hoerl, A. E., Kennard, R. W., 1970. Ridge Regression: Biased Estimation for 388 Nonorthogonal Problems. Technometrics 12 (1), 55-67.

389 Joachims, T., 1998. Text categorization with support vector machines: learning 390 with many relevant features. In: Proceedings of the 10th European Conference 391 on Machine Learning (ECML). Springer Verlag, Heidelberg, DE, pp. 137-142. 392 Joachims, T., 2002. Learning to Classify Text Using Support Vector Machines: 393 Methods, Theory and Algorithms. Kluwer Academic Publishers, Norwell, 394 MA, USA.

395 Jones, K. S., 1988. A statistical interpretation of term specificity and its appli396 cation in retrieval. Document retrieval systems, 132-142.

397 le Cessie, S., van Houwelingen, J. C., 1992. Ridge Estimators in Logistic Re398 gression. Applied Statistics 41 (1), 191-201.

399 Mccullagh, P., Nelder, J. A., 1989. Generalized Linear Models. Chapman \& 400 Hall, London, UK.

401 Minka, T. P., 2003. A Comparison of Numerical Optimizers for Logistic Regres402 sion. Tech. rep., Dept. of Statistics, Carnegie Mellon University.

403 Porter, M. F., July 1980. An algorithm for suffix stripping. Program 14 (3).

404 Salton, G., Wong, A., Yang, C. S., 1975. A Vector Space Model for automatic 405 indexing. Communications of the ACM 18 (11), 613-620.

406 Tibshirani, R., 1994. Regression Shrinkage and Selection Via the Lasso. Journal 407 of the Royal Statistical Society, Series B 58, 267-288.

408 van Rijsbergen, C. J., 1979. Information retrieval, 2nd Edition. Butterworths, 409 London, UK. 
410 Vapnik, V. N., 1995. The nature of statistical learning theory. Springer-Verlag 411 New York, Inc.

412 Zhang, T., Oles, F. J., 2001. Text Categorization Based on Regularized Linear 413 Classification Methods. Information Retrieval 4, 5-31.

414 Zhao, P., Yu, B., 2006. On Model Selection Consistency of Lasso. The Journal 415 of Machine Learning Research 7, 2541-2563.

416 Zou, H., Hastie, T., 2005. Regularization and variable selection via the elastic 417 net. Journal Of The Royal Statistical Society Series B 67 (2), 301-320. 